\title{
Adipose tissue is less responsive to food restriction anti-inflammatory effects than liver, muscle, and brain in mice
}

\author{
M.M. Antunes ${ }^{1 *}$, C.B. de Almeida-Souza ${ }^{1 *}$, G. Godoy ${ }^{1}$, A.R. Crisma ${ }^{2}$, L.N. Masi ${ }^{3}$, R. Curi ${ }^{3}$ \\ and R.B. Bazotte ${ }^{1}$ \\ ${ }^{1}$ Departamento de Farmacologia e Terapêutica, Universidade Estadual de Maringá, Maringá, PR, Brasil \\ ${ }^{2}$ Departamento de Análises Clínicas, Universidade Federal do Paraná, Curitiba, PR, Brasi \\ ${ }^{3}$ Programa de Pós-Graduação Interdisciplinar em Ciências da Saúde, Universidade Cruzeiro do Sul, São Paulo, SP, Brasil
}

\begin{abstract}
High caloric intake promotes chronic inflammation, insulin resistance, and chronic diseases such as type-2 diabetes, which may be prevented by food restriction (FR). The effect of FR on expression of pro-inflammatory and anti-inflammatory genes in adipose tissue, liver, muscle, and brain was compared. Male Swiss mice were submitted to FR (FR group) or had free access to food (control group) during 56 days. The liver, gastrocnemius muscle, brain, and epididymal white adipose tissue (WAT) were collected for analysis of gene expressions. FR attenuated inflammation in the liver, brain, and gastrocnemius muscle but did not markedly change inflammatory gene expression in epididymal WAT. We concluded that adipose tissue was less responsive to FR in terms of gene expression of pro-inflammatory and anti-inflammatory genes.
\end{abstract}

Key words: Caloric restriction; Pro-inflammatory cytokines; Anti-inflammatory cytokines; Acetyl-CoA carboxylase; Disease prevention

\section{Introduction}

Inflammation in the tissues has been associated with excessive caloric intake and sedentarism (1). Prolonged periods of inflammation may cause diseases such as atherosclerosis, insulin resistance, type-2 diabetes, liver steatosis, and neurological disorders. These chronic diseases exhibit marked inflammation in the liver, skeletal muscle, brain, and adipose tissue $(2,3)$.

One effective strategy to prevent inflammation induced by high caloric diet is food restriction (FR) (4). FR has been recommended to reduce inflammation in the liver, brain, muscle, and white adipose tissue (WAT) $(5,6)$.

In spite of this, to clarify the mechanisms involved in the prevention of inflammation by FR more studies are required. For instance, the following question remains unanswered: does attenuation of the inflammation state induced by FR vary depending on the tissue?

In order to address this question, the effect of FR on expression of pro-inflammatory and anti-inflammatory genes in the liver, gastrocnemius muscle, brain, and WAT was compared.

\section{Material and Methods}

\section{Animals and treatments}

Male Swiss mice (Mus musculus) weighing approximately $35 \mathrm{~g}$ were used in the experiments. The animals were housed in a room with a controlled temperature of $23^{\circ} \mathrm{C}$ and an automatically controlled photoperiod (12-h light/12-h dark). The Animal Ethics Committee of State University of Maringá approved the experimental protocol (1067160216/CEUA) used in accordance with the international law on protection and use of animals.

Mice were randomly divided into two groups and were allocated one per cage. The control group ( $C$ group) had free access to food (daily ingestion about $33.3 \mathrm{kcal}$ ), whereas the FR group received $22.8 \mathrm{kcal} /$ day $(31.5 \%$ lower caloric intake compared to the $\mathrm{C}$ group). Food was provided daily to the FR group at 9 a.m. Body weight was determined at day zero and weekly during the 56-day caloric restriction for all mice. During this period, all animals had free access to water.

Correspondence: R.B. Bazotte: <rbbazotte@gmail.com>

*These authors contributed equally to this study. 
All animals received a purified diet for laboratory rodents as proposed by the American Institute of Nutrition for maintenance of laboratory adult rodents (AIN-M-93). The diet composition was described in our previous publication (2).

After 56 days of caloric restriction or free-fed diets, all animals were fasted for $15 \mathrm{~h}$ and killed by decapitation. Blood was collected for measurements of serum glucose, triacylglycerol, and cholesterol concentrations according to the manufacturer's instructions (Labtest ${ }^{\mathbb{R}}$, Brazil). The liver, gastrocnemius muscle, brain, and epididymal WAT were quickly and carefully removed, weighed, frozen in liquid nitrogen, and stored at $-80^{\circ} \mathrm{C}$ until gene expression analysis was performed.

\section{Gene expression analysis}

Expressions of $\mathrm{F} 4 / 80$, interleukin $1 \beta$ (IL-1 $1 \beta$ ), tumor necrosis factor alpha (TNF- $\alpha$ ), IL-6, and IL-10 genes were evaluated in the liver, gastrocnemius muscle, brain, and epididymal WAT.

Expressions of collagen type I and acetyl-CoA carboxylase 1 (ACC1) were measured in the liver while IL-4 was measured in gastrocnemius muscle. Expressions of cyclooxygenase-2 (Cox2), inducible nitric oxide synthase (iNOS), and Ox42 Itgam were measured in the brain.

Total RNA was extracted from liver $(20 \mathrm{mg})$, brain (20 mg), gastrocnemius muscle (20 mg), and epididymal WAT (100 mg) using Trizol reagent (Invitrogen Life Technologies, USA). RNA was quantified by spectrophotometry in a Nano Drop 2000 (ThermoScientific, Uniscience, Brazil) and the purity degree was determined by the $260 /$ $280 \mathrm{~nm}$ ratio.
Reverse transcription to cDNA was performed using the High-Capacity cDNA kit (Applied Biosystems, USA). Real-time PCR (RT-PCR) using SYBR Green as the fluorescent dye (Invitrogen Life Technologies) was used to measure gene expression. Gene expression was estimated using the comparative $\mathrm{Ct}$ method ( $\mathrm{Ct}=$ threshold cycle, the cycle number at which the PCR product reaches the detection threshold), considering the expression of $\beta 2$-microglobulin gene $(\beta 2 \mathrm{~m})$ as the standard reference gene. The relative amounts of each transcript were analyzed using the $2-\triangle \mathrm{C}(\mathrm{t})$ method. The primer sequences were: F4/80, NM_010130.4, sense CCTGAACATGCAA CCTGCCAC, antisense GGGCATGAGCAGBCTGTAGG ATC; Type I collagen, NM_009931.2, sense CTCTATGTC CAAGGCAACGAG, antisense TCACAAACCGCACACCTG; ACC1, NM_133360.2, sense GAGAGGGGTCAAGTCCT TCC, antisense AAAACATCCACTTCCACACACGA; IL-6, NM_001314054.1, sense GGTAGCATCCATCATTTCTT TG, antisense CGGAGAGGAGACTTCACAAGAG; IL-1 $\beta$, NM_008361.4, sense GGCAGCTACCTGTGTCTTTCCC, antisense ATATGGGTCCGACAGCACGAG; TNF- $\alpha$, NM 001278601.1, sense TCTTCTCATTCCTG CTTGTGGC, antisense CACTTGGTGGTTTGCTACGACG; IL-10, NM 010548.2, sense TGCCAAGCCTTATCGGAAATG, antisense AAATCGATGACAGCGCCTCAG; IL-4, NM_021 283.2, sense CCATCTGTGGTGTTCTTCGTTGCTG, antisense ATCCACGGATGCGACA; Cox 2, YP_0016867 01.1, sense AACATCCCCTT CCTGCGAAG, antisense AAGTCCACTCCATGGCCCAG; Itgam, NM_001082960.1, sense TAATGACTCTGCGTTTGCCCTG, antisense ATT GGAGCTGCCCACAATGAG; iNOS, NM_001313921.1, sense CGGCAAACCCAAGGTCTACG, antisense CACCT GCTCCTCGCTCAAGTTC; $\beta 2 \mathrm{M}$, NM_009735.3, sense

Table 1. Body weight, body weight gain, and gastrocnemius muscle, liver, brain, and epididymal white adipose tissue (WAT) weights, and blood levels of glucose, triacylglycerol, and cholesterol.

\begin{tabular}{|c|c|c|}
\hline & Control group & Food restriction group \\
\hline Body weight $(\mathrm{g})$ & $50.50 \pm 1.78$ & $37.10 \pm 0.73^{*}$ \\
\hline Body weight gain $(\mathrm{g})$ & $19.00 \pm 1.65$ & $5.50 \pm 0.94^{*}$ \\
\hline Gastrocnemius muscle weight $(\mathrm{g})$ & $0.47 \pm 0.01$ & $0.41 \pm 0.01^{*}$ \\
\hline Gastrocnemius muscle weight ( $\mathrm{g} / 100 \mathrm{~g}$ body weight) & $0.95 \pm 0.03$ & $1.11 \pm 0.03^{*}$ \\
\hline Liver weight $(\mathrm{g})$ & $1.61 \pm 0.06$ & $1.45 \pm 0.04$ \\
\hline Liver weight ( $\mathrm{g} / 100 \mathrm{~g}$ body weight) & $3.20 \pm 0.09$ & $3.92 \pm 0.12^{*}$ \\
\hline Brain weight $(\mathrm{g})$ & $0.30 \pm 0.01$ & $0.32 \pm 0.01$ \\
\hline Brain weight/Body weight (g/100 g body weight) & $0.67 \pm 0.02$ & $0.87 \pm 0.02^{*}$ \\
\hline Epididymal WAT weight $(\mathrm{g})$ & $1.94 \pm 0.19$ & $1.33 \pm 0.09^{*}$ \\
\hline Epididymal WAT weight ( $\mathrm{g} / 100 \mathrm{~g}$ body weight) & $3.79 \pm 0.26$ & $3.56 \pm 0.20$ \\
\hline Glucose $(\mathrm{mg} / \mathrm{dL})$ & $112.10 \pm 5.39$ & $91.47 \pm 2.32^{*}$ \\
\hline Triacylglycerol (mg/dL) & $69.55 \pm 6.11$ & $43.11 \pm 2.54^{*}$ \\
\hline Cholesterol (mg/dL) & $192.70 \pm 9.79$ & $160.80 \pm 6.06^{*}$ \\
\hline
\end{tabular}

Data are reported as means $\pm S E(n=10)$. ${ }^{*}<0.05$ compared with the Control group (Student's $t$-test for unpaired samples). 


\section{AATGTGAGGCGGGTGGAACTG, antisense CATGGCTC} GCTCGGTGACC.

\section{Statistical analysis}

Data are reported as means \pm SE. Student's $t$-test was used to assess differences between means with the GraphPad Prism Version 5.0 software (GraphPad Software, USA).

\section{Results}

Body weight gain was slower in the FR group $(\mathrm{P}<$ 0.05). After 56 days of caloric restriction, the FR group had lower body mass and body weight gain than $\mathrm{C}$ group $(\mathrm{P}<0.05)$.

The FR had lower gastrocnemius muscle weight and epididymal WAT weight $(P<0.05)$. However, the weight of the liver and brain were not different between the groups. Blood levels of glucose, triacylglycerol, and cholesterol were also lower in the FR group $(P<0.05)$ (Table 1).

The liver relative ACC1 mRNA expressions (means \pm SE of 8-10 mice per group) were $3.23 \pm 1.48$ for the $C$ group and $23.25 \pm 8.23$, for FR group $(P<0.05)$.

The FR group had lower $(P<0.05)$ gene expression of $F 4 / 80$ in the liver (Figure $1 A)$ and higher $(P<0.05)$ gene expression of IL-10 and IL-4 in the gastrocnemius muscle (Figure 1B). The brain of FR mice had lower iNOS and TNF- $\alpha$ gene expressions than $C$ group $(P<0.05$, Figure $1 \mathrm{C}$ ). Epididymal WAT did not exhibit any difference between groups for all genes evaluated (Figure 1D).

\section{Discussion}

FR attenuated inflammation in the liver, brain, and gastrocnemius muscle but did not markedly change inflammatory gene expression in epididymal WAT.
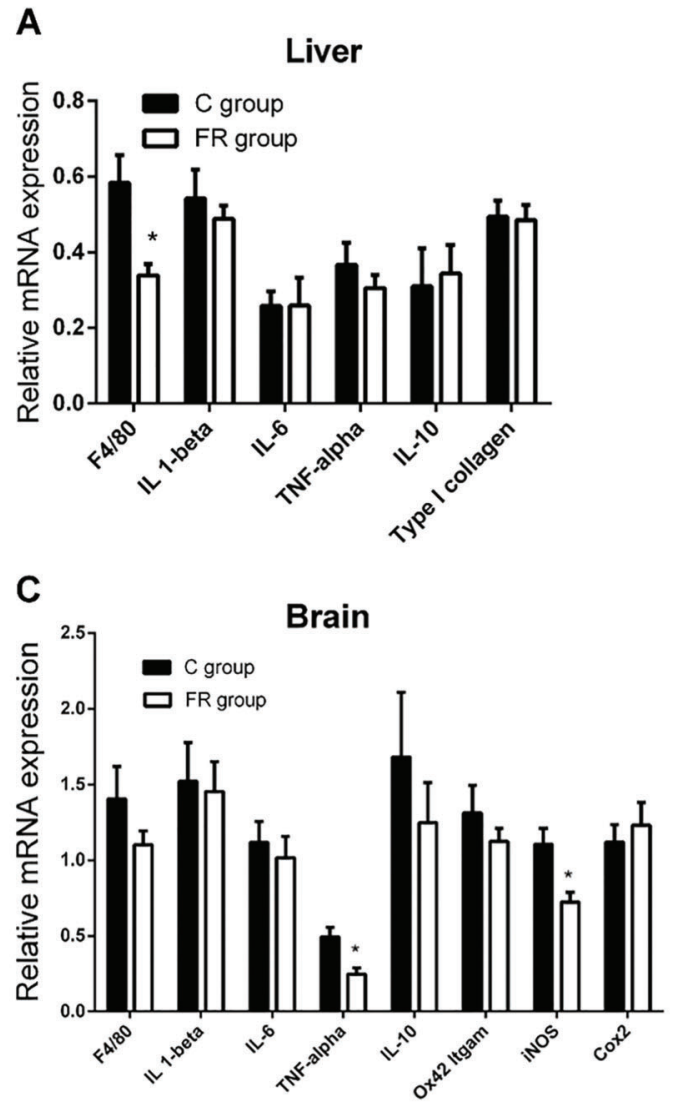
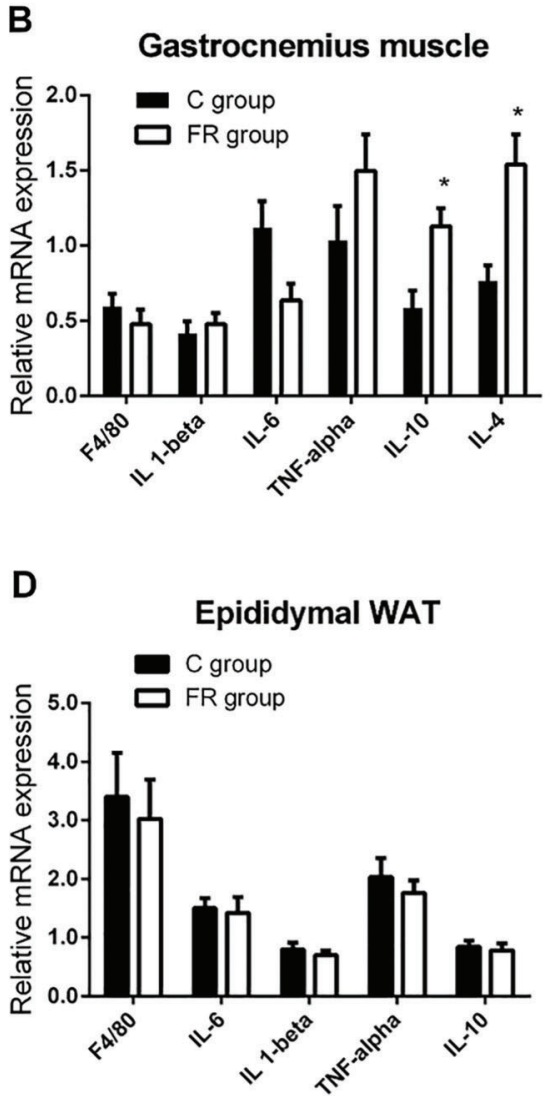

Figure 1. Relative mRNA expression in liver (A), gastrocnemius muscle (B), brain (C), and epididymal white adipose tissue (WAT) (D) in free-fed (C group) or food-restricted mice (FR group). Cox 2: cyclooxygenase 2; IL: interleukin; iNOS: inducible nitric oxide synthase; Ox42 itgam: integrin subunit; TNF-alpha: tumor necrosis factor-alpha. The mRNA expression was determined by RT-PCR. $\beta 2$-microglobulin $(\beta 2 \mathrm{~m})$ was used as housekeeping gene. The relative amounts of each transcript were analyzed using the $2-\Delta \mathrm{C}(\mathrm{t})$ method. Data are reported as means $\pm \mathrm{SE}(\mathrm{n}=8-10) .{ }^{*} \mathrm{P}<0.05$ compared with the $\mathrm{C}$ group (Student's $t$-test for unpaired samples). 
Liver ACC1 expression was seven-fold higher in the FR group. The higher expression of ACC1 is a metabolic adaptation and indicates enhanced capacity of de novo synthesis of fatty acids and increased potential for lipid accumulation during food restriction $(7,8)$.

$\mathrm{F} 4 / 80$, IL-1 $1 \beta, \mathrm{IL}-6, \mathrm{TNF}-\alpha$, and IL-10 gene expression were analyzed in all tissues for two reasons. First, we previously reported changes in gene expression of these five genes in the liver of $C$ group indicating an inflammatory state (2). Second, F4/80, IL-1 $\beta$, IL-6, and TNF- $\alpha$ are biomarkers of inflammatory states (1-9), while IL-10 represents one of the major anti-inflammatory cytokines (10). Moreover, these cytokines have profound metabolic effects creating complex networks of interactions between cells and tissues, leading to multiple inflammatory cascades (1-6). Additional specific genes better represent inflammation in different tissues. In the liver, for example, type I collagen indicates fibrosis and chronic liver injury (3). In the brain, Ox42 itgam, iNOS, and Cox2 genes are well established inflammatory biomarkers $(11,12)$, and IL-10 and IL-4 have a pivotal role as anti-inflammatory cytokines (10).

The liver from FR mice exhibited decreased F4/80 expression, which is one of the most specific cell-surface markers of macrophage and thus, a marker of inflammation (2). The lower expression of F4/80 in the FR group indicated less macrophage infiltration in the liver and therefore attenuation of inflammation and fatty liver disease (9).

Gastrocnemius muscle exhibited increased expressions of anti-inflammatory cytokines IL-10 and IL-4 due to FR. IL-10 is an important regulator of IL-6, TNF- $\alpha$, and IL-1 $\beta$ levels whereas IL-4 plays an important role in skeletal muscle regeneration (10).

\section{References}

1. Hotamisligil GS. Inflammation and metabolic disorders. Nature 2006; 444: 860-867, doi: 10.1038/nature05485.

2. da Silva-Santi L, Antunes MM, Caparroz-Assef SM, Carbonera $F$, Masi $L N$, Curi $R$, et al. Liver fatty acid composition and inflammation in mice fed with high-carbohydrate diet or high-fat diet. Nutrients 2016; 8: E682, doi: 10.3390/nu811 0682.

3. Masi LN, Martins AR, Crisma AR, do Amaral CL, Davanso MR, Serdan TDA, et al. Combination of a high-fat diet with sweetened condensed milk exacerbates inflammation and insulin resistance induced by each separately in mice. Sci Rep 2017; 7: 3937, doi: 10.1038/s41598-017-04308-1.

4. de Almeida-Souza CB, Antunes MM, Godoy G, Schamber CR, da Silva MARCP, Bazotte RB. Interleukin-12 as a biomarker of the beneficial effects of food restriction in mice receiving high fat diet or high carbohydrate diet. Braz J Med Biol Res 2018; 51: e7900, doi: 10.1590/1414431X20187900.

5. Ye J, Keller JN. Regulation of energy metabolism by inflammation: A feedback response in obesity and calorie restriction. Aging 2010; 2: 361-368, doi: 10.18632/aging. 100155.
FR prevents brain diseases (11). This beneficial effect of FR includes metabolic reprogramming and changes in the levels of cytokines that regulate neuroinflammation (12). In agreement with this statement, we observed lower brain iNOS and TNF- $\alpha$ expressions.

In contrast with other tissues, epididymal WAT did not exhibit any difference in the expressions of the genes measured. The absence of changes in inflammatory gene expressions in WAT reported in this study, however, does not discard the possibility that differences may occur with prolonged periods of FR or other types of diets. For example, nine-month FR, as well high-fat diet models associated with FR in mice down-regulates expressions of inflammatory genes in WAT $(13,14)$.

Taken together, our results and findings reported by others (13-15) suggest that the attenuation of the inflammation state induced by FR not only is time-dependent but also varies with the tissue.

The clinical relevance of this investigation is that the FR anti-inflammatory effects could be progressive, and prolonged periods might be necessary to reach the maximum benefits of food restriction.

\section{Acknowledgments}

We are grateful to Christiano Rodrigues Schamber and Carlos Eduardo de Oliveira for their technical assistance. This research was supported by the following research agencies: CNPq/PRONEX/Araucária Foundation, FAPESP (grant number 2016/15766-7), CAPES, and Guggenheim Foundation [grant number 249/2013].

6. Huang P, Li S, Shao M, Qi Q, Zhao F, You J, et al. Calorie restriction and endurance exercise share potent antiinflammatory function in adipose tissues in ameliorating diet-induced obesity and insulin resistance in mice. Nutr Metab 2010; 7: 59, doi: 10.1186/1743-7075-7-59.

7. Bruss MD, Khambatta CF, Ruby MA, Aggarwal I, Hellerstein MK. Calorie restriction increases fatty acid synthesis and whole body fat oxidation rates. Am J Physiol Endocrinol Metab 2010; 298: E108-E116, doi: 10.1152/ajpendo.00524. 2009.

8. Bazotte RB, Batista MR, Curi R. Meal-feeding scheme: twenty years of research in Brazil. Braz J Med Biol Res 2000; 33: 985-991, doi: 10.1590/S0100-879X2000000900001.

9. Huang W, Metlakunta A, Dedousis N, Zhang P, Sipula I, Dube JJ, et al. Depletion of liver kupffer cells prevents the development of diet-induced hepatic steatosis and insulin resistance. Diabetes 2010; 59: 347-357, doi: 10.2337/db090016.

10. Hong E, Ko HJ, Cho YR, Kim HJ, Ma Z, Yu TY, et al. Interleukin-10 Prevents diet-induced insulin resistance skeletal muscle. Diabetes. 2009; 58: 2525-2535, doi: 10.2337/ db08-1261. 
11. Loncarevic-vasiljkovic N, Pesic V, Todorovic S, Popic J, Smiljanic K, Milanovic $D$, et al. Caloric restriction suppresses microglial activation and prevents neuroapoptosis following cortical injury in rats. Plos One 2012; 7: e37215, doi: 10.1371/ journal.pone.0037215.

12. Kaminska B, Mota M, Pizzi M. Signal transduction and epigenetic mechanisms in the control of microglia activation during neuroinflammation. Biochim Biophys Acta 2016; 3: 339-351, doi: 10.1016/j.bbadis.2015.10.026

13. Higami Y, Barger JL, Page GP, Allison DB, Smith SR, Prolla $\mathrm{TA}$, et al. Energy restriction lowers the expression of genes linked to inflammation, the cytoskeleton, the extracellular matrix, and angiogenesis in mouse adipose tissue. $J$ Nutr 2006; 136: 343-352, doi: 10.1093/jn/136.2.343.

14. Lijnen HR, Hul M Van, Hemmeryckx B. Caloric restriction improves coagulation and inflammation profile in obese mice. Thromb Res 2012; 129: 74-79, doi: 10.1016/j.thromres. 2011.05.023.

15. Araújo EP, De Souza CT, Ueno M, Cintra DE, Bertolo MB, Carvalheira JB, et al. Infliximab restores glucose homeostasis in an animal model of diet-induced obesity and diabetes. Endocrinology 2007; 148: 5991-5997, doi: 10.1210/en.2007-0132. 